\title{
ANALYSIS AND RESULTS OF THE INDUSTRIAL PRODUCTION OF THE SUPERCONDUCTING Nb/Cu CAVITIES FOR THE LEP 2 PROJECT
}

\author{
E. Chiaveri, C. Benvenuti, R. Cosso, D. Lacarrere, K.M. Schirm, M. Taufer, W. Weingarten \\ CERN, 1211 Geneva 23, Switzerland
}

\begin{abstract}
For the energy upgrade of the Large Electron Positron Collider at CERN, 216 RF superconducting cavities were ordered from three European industrial firms (Ansaldo,Cerca,Siemens/Accel) at the beginning of 1991. These cavities are made of copper $(\mathrm{Cu})$, internally coated with niobium $(\mathrm{Nb})$ according to a procedure developed at CERN. Up to now about 147 of these cavities fulfilling the specifications have been produced. The large-scale statistics available and the use of dedicated analytical and optical inspection techniques shed new light on the relationship between production procedures, niobium film properties and cavity performance. An overview of this subject is presented, together with some significant trends and results.
\end{abstract}

\section{INTRODUCTION}

Niobium sputter coating of four-cell $350 \mathrm{MHz}$ cavities built from OFE copper sheet material has been chosen at CERN for series production. This technology presents some interesting advantages in terms of thermal stability, costs and future potential [1]. Until now 147 "bare" cavities meeting the technical specification $\left(\mathrm{Q}=3.4 \times 10^{9}\right.$ at $\mathrm{E}=6 \mathrm{MV} / \mathrm{m}$ at $4.5 \mathrm{~K}$ ) have been produced representing $65 \%$ of the total present contractual number (216). After a period of about one year for the transfer of technology, the three firms started to produce one cavity per week according to the planning. However, we were faced with a problem of reproducibility of the coating process related to the very delicate procedures which require a high level of quality control.

The cavity performance is sometimes limited by local defects (up to several $\mathrm{mm}$ in size) of the $\mathrm{Nb}$ layer. Major defects like poor bonding (blister) of small $\mathrm{Nb}$ areas (Fig. 1) are usually detected as "hot spots" in the temperature maps [2] of RF tests. Investigations of such defective layers by optical [3] and surface analytical tools [4,5] have been launched in order to clarify the nature and origin of various types of defect and also of other minor irregularities that can occur in considerable numbers without being detected in temperature maps. After inspection, these cavities must have the defective $\mathrm{Nb}$ layer chemically removed at CERN and are then returned to the manufacturer for a second or even a third $\mathrm{Nb}$ coating.

\section{ANALYSIS OF THE CAVITY SURFACE}

Cavities that are rejected after the RF measurements are systematically investigated by means of a video camera setup on a computer controlled optical inspection bench [3]. Pictures of surface defects and irregularities (fig.1 and 2) are taken at different magnification together with the position coordinates.

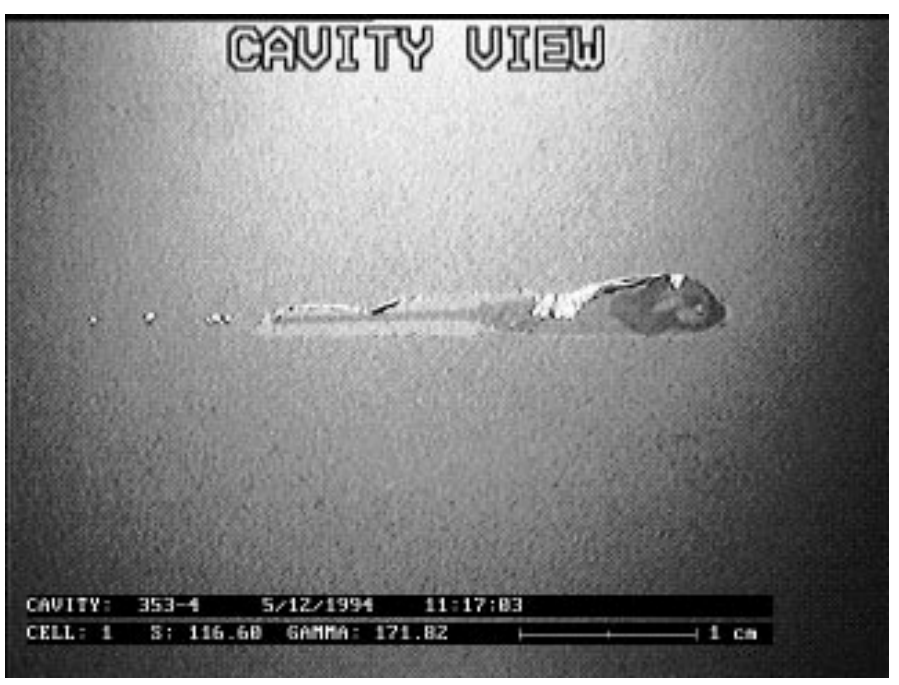

Fig.1: Major defect on the inner cavity surface $(\sim 2 \mathrm{~cm})$.

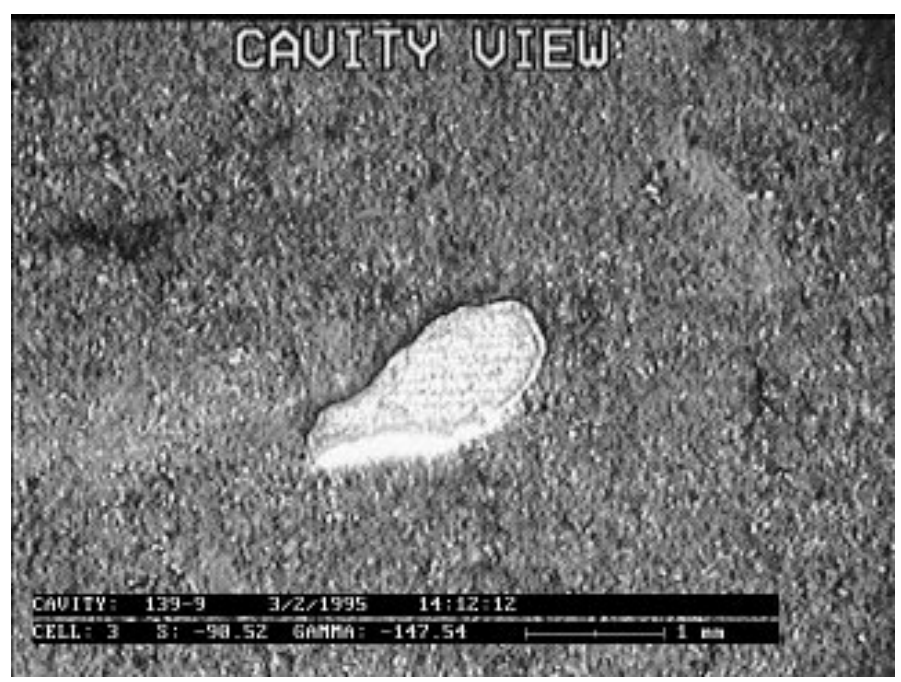

Fig.2: Inner cavity surface with $1.5 \mathrm{~mm}$ long irregularity.

The results of the optical inspection and the temperature maps provide in most cases an explanation for the inadequate performance. However further specific surface 
analysis is necessary for understanding the nature of these defects. In view of this, a computer controlled surface analysis instrument, incorporating Secondary Electron Microscopy (SEM) imaging, Auger Electron Spectroscopy (AES) and Scanning Auger Mapping (SAM) has been designed and built at CERN [4,5]. This diagnostic tool provides both topographical information and elemental composition of surface defects.

Some characteristic defects of the $\mathrm{Nb}$ film were observed during industrial cavities investigation carried out so far. For example, corresponding to defective areas located by SED imaging, the $\mathrm{Nb}$ film was often found to be very thin or missing by AES analysis; the $\mathrm{Cu}$ substrate was in some cases highly contaminated, mainly with $\mathrm{C}$, and also with $\mathrm{S}$, $\mathrm{Cl}, \mathrm{N}$; in other cases, particles containing $\mathrm{Fe}$ and $\mathrm{Ni}$ were detected, embedded in the $\mathrm{Cu}$ substrate.

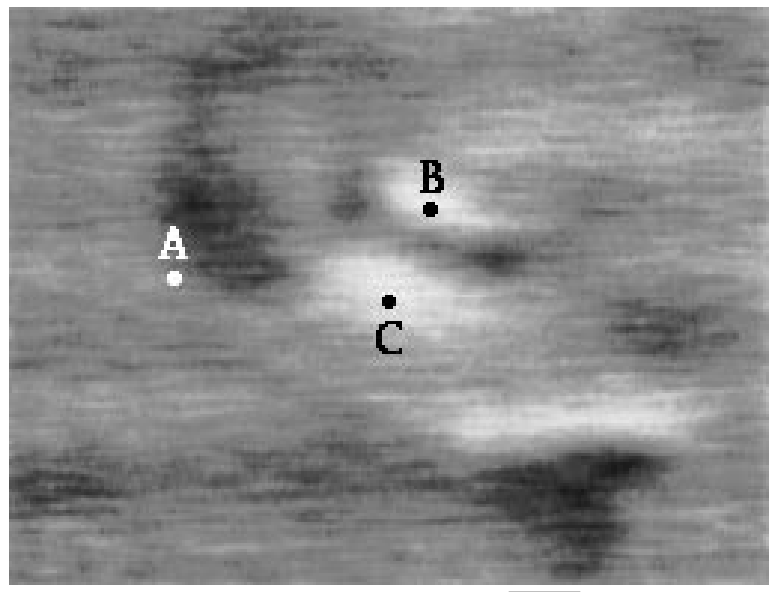

Fig.3a: Secondary electron image $\overline{100} \mu \mathrm{m}$

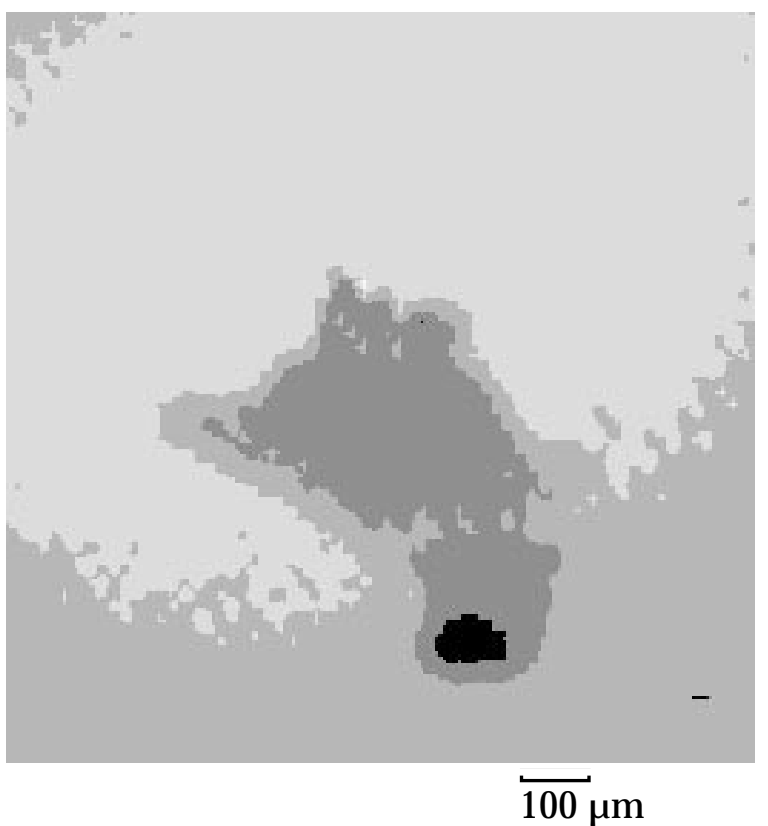

Fig.3b: Scanning Auger O map (darker areas correspond to lower concentration of $\mathrm{O}$ )
In figure 3 an example of characterization of a defective area is shown. A Secondary Electron Image of the defective area (3a), its associated O elemental map (3b), and related Auger electron spectra (3c) are presented. Since the surface of the film consists of $\mathrm{Nb}$ oxidised mainly in the form $\mathrm{Nb}_{2} \mathrm{O}_{5}$ [6], corresponding to an atomic concentration of oxygen $\mathrm{X}_{\mathrm{O}} \approx$ 0.7 , it can be concluded that the light area in fig. $3 \mathrm{~b}$ corresponds to a region where the $\mathrm{Nb}$ film is present and the dark area to a region where it is either very thin or missing. The black spot at the bottom of the picture corresponds to the highest concentration observed in this area. This conclusion is supported by the Auger spectra shown in $3 \mathrm{c}$, corresponding to points $\mathrm{A}, \mathrm{B}$ and $\mathrm{C}$ in the SED image (3a). In spectrum A no signal from the substrate is detected, whilst in B the copper 'fingerprint' is clearly visible; in $\mathrm{C}$, copper is still present but the main contribution to the spectrum is given by the carbon line.

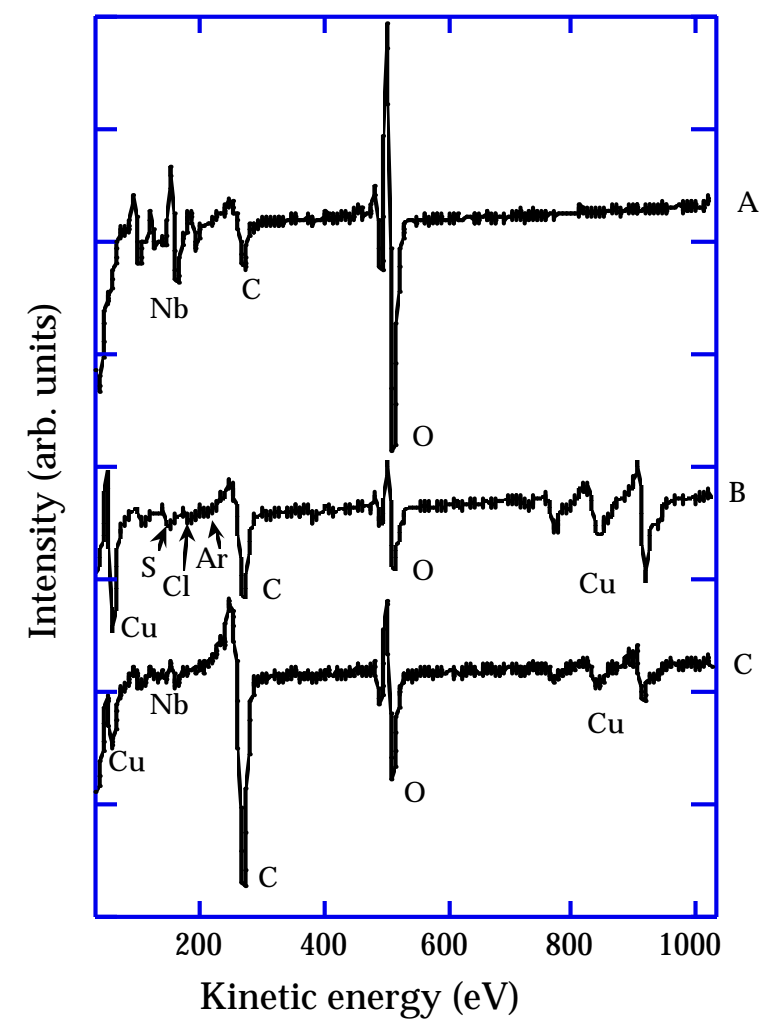

Fig.3c: Auger spectra taken at points A, B and C

\section{RESULTS AND DISCUSSION}

It seems clear by now that we have to distinguish between defects originating from the production of the raw $\mathrm{Cu}$ cavities and accidents happening to them afterwards before coating, e.g. during the chemistry/drying cycles. Some types of defect are company-specific, others have a more general nature, but all of them can lead to noticeable peeling of the active layer. It was recently found that the probability of such defects occuring is reduced by applying additional successive cycles of electrolytical and chemical 
polishing to the copper. Removing the copper surface to an increased depth (more than $150 \mu \mathrm{m}$ total) not only reduces the risk of chemical retention in pits [7] but also eliminates traces of the mechanical polishing done after the electronbeam welding assembly.

For cavities without noticeable defects we have identified two process parameters in the industrial production that could be highly relevant for the RF performance:

i) The vacuum quality before the $\mathrm{Nb}$ coating

ii) The temperature chosen for baking and sputtering.

These parameters are interconnected since the bake-out protocol also determines the vacuum quality. Various possible incidents in the delicate manufacturing process preceding the coating can change the residual gas composition as indicated by mass spectrometry and the attainable pressure limit in the cavity (usually $<10^{-9}$ Torr). Consequences range from a decreased $\mathrm{Q}$ value at low field by the getter pumping effect of light gases during $\mathrm{Nb}$ deposit [1] to an insufficient $\mathrm{Nb}$ adhesion in presence of organic residues. The choice of the cavity temperatures for baking and sputtering determines not only the diffusion and outgassing rates but also the growth properties of the $\mathrm{Nb}$ layer and structural aspects of the copper substrate and their influence on its mechanical properties. In view of these partly conflicting aspects the best compromise appears to be the following: baking the cavities at $150^{\circ} \mathrm{C}$ for $24 \mathrm{~h}$ and coating them at cell temperatures strictly limited to $\leq 200^{\circ} \mathrm{C}$ (temperature maxima usually $180^{\circ} \mathrm{C} \pm 10^{\circ} \mathrm{C}$ ). Respecting all the criteria mentioned above has resulted, at all three companies, in cavity performances well above the specification requirements (Fig.4) and considerably increased acceptance rates of cavities with only one $\mathrm{Nb}$ coating (Fig.5).

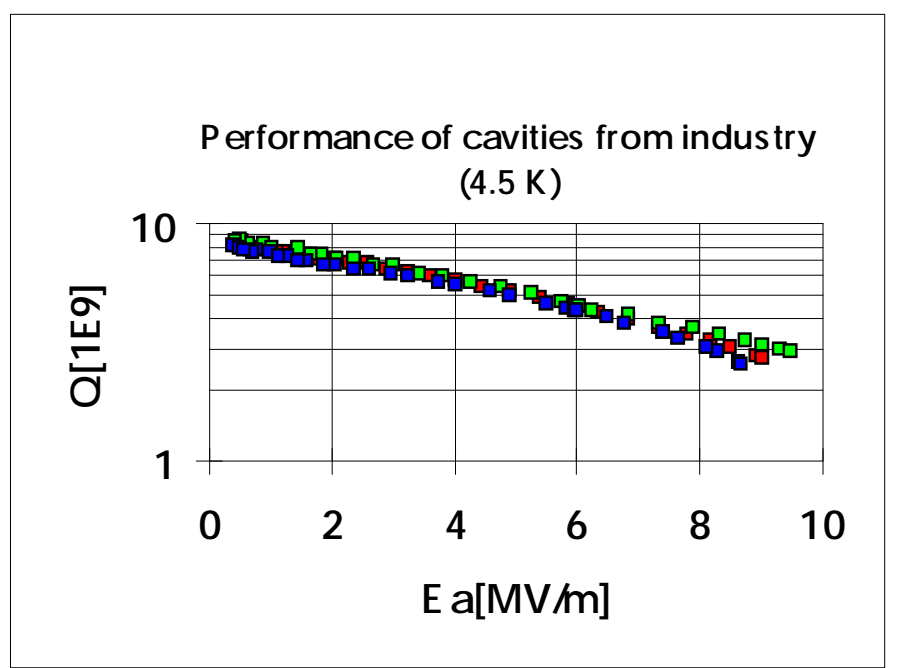

Fig.4: Recently obtained cavity performance for different manufacturers.

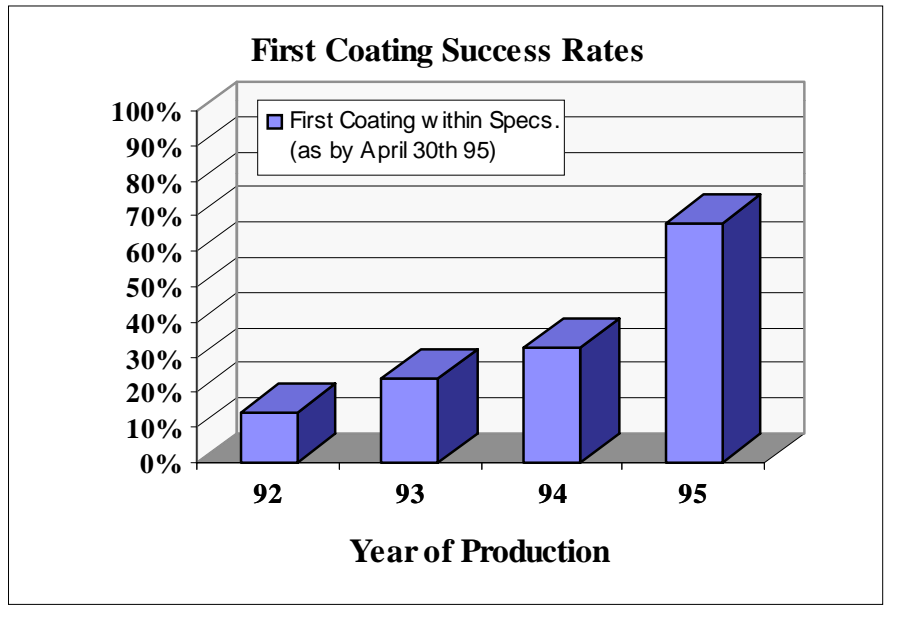

Fig.5: Total first coating success rates in industry

\section{CONCLUSION}

Superconducting $\mathrm{Nb} / \mathrm{Cu}$ cavity production technology has been improved in the course of the large-scale series manufacturing of $350 \mathrm{MHz}$ cavities. Statistical survey of the correlation between key process parameters and performance tests as well as the use of dedicated analysis tools have helped to improve our understanding of the fabrication process and to modify it effectively. Q-factors are now consistenly better than specifications (by $25 \%$ on average) but the random occurrence of surface defects still limits the coating success rate. A rigorous quality control is mandatory.

\section{ACKNOWLEDGEMENTS}

The dynamism and the technical competence of R.Hanni, E.Magnani, A.Scharding have been instrumental in the successful advancement of the project. We also would like to thank B.Thony for his useful help on the optical analysis. We appreciate the effort of G.Bressani for the set-up of the optical inspection bench. Many thanks to D.Boussard for his critical remarks.

\section{REFERENCES}

[1] C. Benvenuti et al.; Physica B 197 (1994) 72

[2] Ph.Bernard et al. Nucl. Instr. and Meth. 190 (1981) 257-282

[3] G.Bressani et al. paper in preparation

[4] D. Lacarrere et al., Proceedings of the 6th workshop on RF Superconductivity, Newport News, Virginia USA, 4-8 Oct. 93; p.702/717

[5] C. Benvenuti et al., submitted to NIM

[6] J. Halbritter, Appl. Phys. A 43 (1987) 1.

[7] S. Calatroni et al., Proceedings of the 6th workshop on RF Superconductivity, Newport News, Virginia USA, 4-8 Oct. 93; p.702/717 
